HUB-EP-96/40

hep-ph/9608223

August 1996

\title{
Extended NJL Model for light and heavy mesons without $q-\bar{q}$ thresholds
}

\author{
Dietmar Ebert*, Thorsten Feldmann* ${ }^{* \ddagger}$ \\ Institut für Physik, Humboldt-Universität zu Berlin, \\ Invalidenstraße 110, D-10115 Berlin, Germany \\ Hugo Reinhardt! \\ Institut für Theoretische Physik, Universität Tübingen, \\ Auf der Morgenstelle 14, D-72076 Tübingen, Germany
}

\begin{abstract}
We consider the NJL model as an effective quark theory to describe the interaction which is responsible for the quark flavor dynamics at intermediate energies. In addition to the usual ultraviolet cut-off which is necessary since the model is non-renormalizable, we also introduce an infrared cut-off which drops off the unknown confinement part of the quark interaction, which is believed to be less important for the flavor dynamics. The infrared cutoff eliminates all $q-\bar{q}$ thresholds, which plague the application of the usual NJL model beyond low-energy pion physics. We apply this two-cut-off prescription to the extended NJL model with chiral and heavy quark symmetries proposed recently by us. We find a satisfactoring description even of the heavy mesons with spin/parity $J^{P}=\left(0^{+}, 1^{+}\right)$. Furthermore, the shape-parameters of the Isgur-Wise function are studied as a function of the residual heavy meson mass.
\end{abstract}

PACS: $12.39 . \mathrm{Ki}$, 12.39.Fe, 12.39. Hg

Keywords: Nambu-Jona-Lasinio Model, Heavy Quark Symmetry, Chiral Symmetry

*Supported by Deutsche Forschungsgemeinschaft under contract Eb 139/1-2.

†Supported by COSY under contract 41170833.

${ }^{\ddagger}$ E-mail: FELDMANN@PHA2.PHYSIK.HU-BERLIN.DE 


\section{Introduction}

The NJL model has proved quite successful in describing the low-energy meson physics [1]. Its successes stem from the fact that it embodies chiral symmetry and its spontaneous breaking. Unfortunately, the model is not renormalizable. This necessitates the introduction of an ultraviolet cut-off $\Lambda$, indicating the range of validity of the model. Futhermore the model does not confine the quarks, and as a consequence the model suffers from unphysical $q-\bar{q}$ thresholds, which severely restrict the applications of the model. The lack of quark confinement in NJL-type models is related to the fact, that the very infrared region of the gluon-mediated quark interactions is not adequately incorporated. In fact, such models are usually constructed to describe the low-energy properties of mesons, which are assumed to be dominated by the quark flavor dynamics at intermediate scales, say between a confinement scale of a few hundred $\mathrm{MeV}$ and a scale of about $1 \mathrm{GeV}$. The success of these models is related to the very fact, that chiral symmetry is dynamically broken just in this energy region, and that the picture of (constituent) quarks interacting with mesons may be a reasonable approximation to strong interactions. Since on the other hand this picture must be wrong for very low scales where confinement becomes definitely important, one better excludes the infrared region explicitly by introducing an IR cut-off as long as one has no definite suitable way of introducing confinement?. We will show that the problems connected with unphysical quark-antiquark thresholds are then automatically removed.

As an illustration we apply the two-cut-off prescription to the recently proposed extended NJL model with both chiral symmetry for the light quarks $m_{q} \ll \Lambda_{Q C D}$ and heavy quark symmetries? for the heavy flavors $m_{Q} \gg \Lambda_{Q C D}$ [8]. There, the usual low-momentum expansion (LME) has been applied to analyze the quark determinant, and reasonable results have been obtained for nearly all heavy meson observables. However, for the slope-parameter of the Isgur-Wise function we found a smaller value than expected, and the heavy meson states with spin/parity $J^{P}=\left(0^{+}, 1^{+}\right)$could not be well described. It seems therefore necessary to study the influence of the external momenta more carefully, which is however not under control as long as the quark model under concern has no explicit implementation of confinement. In this case, for meson momenta larger than the (constituent) quark masses, the decay of a meson into a real quark-antiquark pair becomes kinematically allowed, and one runs into trouble with unphysical imaginary parts due to quarkantiquark thresholds. The LME circumvents this problem; in this way the low-energy theorems in the light meson sector can be recovered (see e.g. [1]), while in the heavy meson sector the LME seems to be insufficient since a smooth dependence on external momenta is not always found, i.e. the LME does not converge for momenta beyond thresholds.

We will first present the regularized NJL model and discuss the physical implications of the infrared cut-off on the spontaneous breaking of chiral symmetry. For this purpose we study the gap-equation that describes the dynamical generation of the constituent quark mass in dependence of the infrared cut-off. Then we apply the regularization method to the extended NJL model with chiral and heavy quark symmetries [8]. The mass spectrum of heavy mesons is found, which indeed develops a smooth dependence on external momenta - even beyond the apparent thresholds - and leads to reasonable predictions. As an application, we finally discuss the slope and curvature of the Isgur-Wise function for finite external momenta and compare to the phenomenological findings.

\footnotetext{
${ }^{1}$ There are non-local extensions of the NJL model (see e.g. [2] and refs. therein) where the thresholds are avoided. However, there the beauty and simplicity of the local NJL model is lost, i.e. one has to deal with integral equations, that must be solved numerically.

${ }^{2}$ see e.g. refs. [3, 4, 5, 6, , 7
} 


\section{Implications of an infrared cut-off in the NJL model}

The NJL model rewritten in bosonic degrees of freedom (see eq. (6) below) leads to an effective meson action where besides meson mass terms the quark determinant $\operatorname{Tr} \ln i \not D$ contributes. Here $i \not D$ is the Dirac operator of the quarks in the presence of meson fields. The real part of the quark determinant is a diverging object. Using a proper-time regularization, this quantity becomes (after continuation to Euclidean space)

$$
\ln |\operatorname{det} i \not D| \rightarrow-\frac{N_{c}}{2} \int_{1 / \Lambda^{2}}^{1 / \mu^{2}} \frac{d s}{s} \int \operatorname{Tr}\left[e^{-s \not D E_{E}^{\dagger} \not D_{E}}\right] .
$$

Here $\Lambda$ is the before-mentioned ultraviolet cut-off. Furthermore we have introduced here also an infrared cut-off $\mu$, which takes into account that our model is not applicable in the very lowenergy regime due to the lack of confinement. This infrared cut-off is the new feature of the present analysis. All previous applications of the NJL model have effectively used the limit $\mu \rightarrow 0$. A simple way to understand that the suggested method indeed removes the quark-thresholds is to consider the simplest Greens function obtained from eq. (1), namely the effective light quark propagator. In Euclidean space one obtains for the denominator

$$
\int_{1 / \Lambda^{2}}^{1 / \mu^{2}} d s \exp \left[-s\left(k_{E}^{2}+m^{2}\right)\right]=\frac{e^{-\frac{k_{E}^{2}+m^{2}}{\Lambda^{2}}}-e^{-\frac{k_{E}^{2}+m^{2}}{\Lambda^{2}}}}{k_{E}^{2}+m^{2}} .
$$

Obviously, the former pole at $-k_{E}^{2}=k^{2}=m^{2}$ is removed by the infrared cut-off. We consider $\mu$ as an adjustable parameter which may be interpreted as the scale up to which the quarks have been integrated out. Especially, at $\mu=\Lambda$ (which is the UV cut-off of the model) the contribution of the quark determinant vanishes by construction, and we recover the original interaction of (current) quarks with non-dynamical meson fields, where chiral symmetry is not (yet) broken. Note that the non-renormalizable NJL model is defined only after the regularization method has been specified, and in this sense the ultraviolet cut-off $\Lambda$ and the infrared cut-off $\mu$ together with the proper-time regularization are parts of the definition of the model.

In the NJL model [1] the spontaneous breaking of chiral symmetry is realized dynamically through a non-vanishing VEV for the scalar field $\Sigma$ (chiral radius) which is determined by the following gap equation

$$
\langle\Sigma\rangle=m=m_{0}+8 m G_{1} I_{1}\left(m^{2}\right), \quad I_{1}\left(m^{2}\right)=\frac{N_{c}}{16 \pi^{2}} m^{2} \Gamma\left[-1, m^{2} / \Lambda^{2}, m^{2} / \mu^{2}\right],
$$

This equation defines the constituent quark mass $m$ as an implicit function of the scale $\mu$ for given values of the remaining model parameters. Here $\Gamma[\alpha, x, y]=\int_{x}^{y} d t \exp (-t) t^{\alpha-1}$ denotes the incomplete Gamma function.

For a numerical discussion we use the coupling constant and the cutoff from ref. [8] as $G_{1}=$ $5.25 \mathrm{GeV}^{-2}$ and $\Lambda=1.25 \mathrm{GeV}$. Fig. 1 shows the solution of the gap equation $m$ as a function of the IR scale $\mu$ in the chiral limit $\left(m_{0}=0\right)$. For scales $\mu<0.3 \mathrm{GeV}$ the order parameter $m$ of the chiral symmetry breaking is almost independent of $\mu$, while it decreases rapidly when $\mu$ exceeds a value of $\mu \approx 0.3 \mathrm{GeV}$ and vanishes at a critical value $\mu_{c}$ given by

$$
\mu_{c}^{2}=\Lambda^{2}-\frac{2 \pi^{2}}{3 G_{1}} \approx(550 \mathrm{MeV})^{2}
$$

\footnotetext{
${ }^{3}$ An exception is ref. 9] where a sharp momentum cut-off was used for both the ultraviolet and infrared regime. However, the authors do not take into account the effect of the external momenta and consequently do not address the question of quark-confinement (In fact they use the limit $\mu, m_{q}, v \cdot p \ll \Lambda$ for a rough estimate).
} 


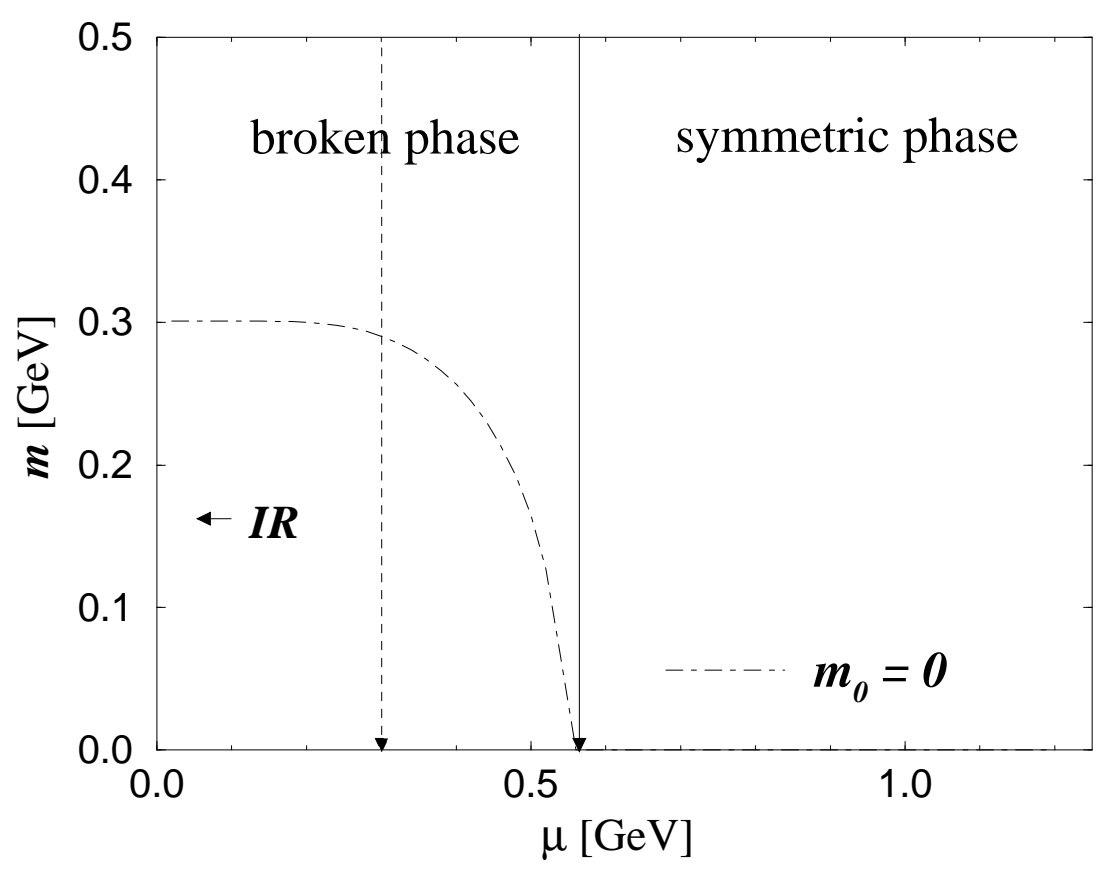

Figure 1: The light quark constituent mass $m$ following from the gap-equation as a function of the scale $\mu$ for the chiral limit $m_{0}=0$. The bold vertical line denotes the separation of the chirally symmetric and chirally broken phase. The dashed vertical line denotes the chosen separation of the IR (confinement) region at a scale $\mu \approx 300 \mathrm{MeV}$.

For $\mu>\mu_{c}$ chiral symmetry is restored. Consequently, in order to include the effect of the spontaneous breaking of chiral symmetry, it is sufficient to consider the quark flavor dynamics down to scales of about $\mu \approx 300 \mathrm{MeV}$. At lower values of $\mu$ one expects essential effects of the IR singular gluon propagator, leading to a modified gap- (or in this case Schwinger-Dyson-) equation and a presumably confined quark propagator. Clearly, this question cannot be addressed within the NJL model and is outside the scope of the present paper.

\section{Extended NJL model}

In [8] we have presented an extension of the NJL model which combines chiral symmetry for light quarks with the heavy quark symmetries. In the heavy mass limit, heavy meson fields are organized in spin symmetry doublets [4]

$$
\begin{array}{ll}
H=\frac{1+\psi}{2}\left\{i \Phi^{5} \gamma_{5}+\Phi^{\mu} \gamma_{\mu}\right\} & , \quad v_{\mu} \Phi^{\mu}=0 \\
K=\frac{1+\psi}{2}\left\{\Phi+i \Phi^{5^{\prime \mu}} \gamma_{\mu} \gamma_{5}\right\} & , v_{\mu} \Phi^{5^{\prime \mu}}=0
\end{array}
$$

with $v^{\mu}$ being the heavy quark velocity. Here $\Phi^{5}$ and $\Phi^{\mu}$ denote the pseudoscalar and vector field of the $J^{P}=\left(0^{-}, 1^{-}\right)$multiplet, $\Phi$ and $\Phi^{5^{\prime \mu}}$ are the heavy scalar and axial vector field of the 
$J^{P}=\left(0^{+}, 1^{+}\right)$multiplet.

After introducing light and heavy meson fields, the lagrangian of the extended NJL model can be rewritten as 8

$$
\begin{aligned}
\mathcal{L}= & -i N_{c} \operatorname{Tr} \ln i D^{r e g}-\frac{1}{4 G_{1}} \operatorname{tr}_{\mathrm{F}}\left[\Sigma^{2}-\widehat{m}_{0}\left(\xi \Sigma \xi+\xi^{\dagger} \Sigma \xi^{\dagger}\right)\right] \\
& +\frac{1}{4 G_{2}} \operatorname{tr}_{\mathrm{F}}\left[\left(V_{\mu}-\mathcal{V}_{\mu}^{\pi}\right)^{2}+\left(A_{\mu}-\mathcal{A}_{\mu}^{\pi}\right)^{2}\right]-\frac{1}{2 G_{3}} \operatorname{Tr}[\psi(\bar{H}+\bar{K})(H+K)],
\end{aligned}
$$

with $i \not \supset$ being the light quark determinant:

$$
i \not D=i \not \partial-\Sigma+Y+\not A \gamma_{5}-(\bar{H}+\bar{K})(i v \cdot \partial)^{-1}(H+K)
$$

which contains the light scalar, vector and axial-vector meson fields $\left(\Sigma, V_{\mu}, A_{\mu}\right)$ and heavy meson fields $(H, K) . N_{c}=3$ is the color factor, $\hat{m}_{0}$ is the current mass matrix of light quark flavors and $G_{1}, G_{2}, G_{3}$ are independent coupling constants of four-quark interactions allowed by chiral and heavy quark symmetries ( $\operatorname{dim} G_{1,2,3}=\operatorname{mass}^{-2}$ ). For the light octet of Goldstone bosons we use the common non-linear representation $\xi=\exp (i \pi / F)$ where $\pi=\pi^{a} \lambda_{F}^{a} / 2$ and $F$ is the bare decay constant. The induced (axial-)vecotr fields $\mathcal{V}_{\mu}^{\pi}=i / 2\left(\xi \partial_{\mu} \xi^{\dagger}+\xi^{\dagger} \partial_{\mu} \xi\right), \mathcal{A}_{\mu}^{\pi}=i / 2\left(\xi \partial_{\mu} \xi^{\dagger}-\xi^{\dagger} \partial_{\mu} \xi\right)$ arise from a chiral rotation of the quark fields. Finally, the light scalar field $\Sigma$ achieves a non-vanishing vacuum expectation value indicating the spontaneous breaking of chiral symmetry (see eq. (3) above). For further details we refer the reader to ref. [8].

For heavy mesons with the residual external momentum $v \cdot p$ the self-energy contribution from the quark loop, together with the constant terms in eq. (6), determines the residual masses $\Delta M=$ $M-m_{Q}$ and the renormalization factors $Z$

$$
\begin{gathered}
\frac{1}{2 G_{3}} \stackrel{!}{=} I_{3}\left(v \cdot p, m^{2}\right)(v \cdot p \pm m)+\left.I_{1}\left(m^{2}\right)\right|_{v p=\Delta M_{H, K}} \\
Z_{H, K}=\left.\left(I_{3}\left(v \cdot p, m^{2}\right)+\frac{\partial}{\partial v \cdot p} I_{3}\left(v \cdot p, m^{2}\right)(v \cdot p \pm m)\right)^{-1}\right|_{v p=\Delta M_{H, K}}
\end{gathered}
$$

where the upper sign $(+)$ is for the $H$-fields and the lower sign $(-)$ for the $K$-fields, indicating the mass splitting of the two parity conjugated states due to the broken chiral symmetry for $m \neq 0$. The function $I_{3}$ which diverges linearly with the UV cutoff $\Lambda$ is represented by the proper-time integral

$$
I_{3}\left(v \cdot p, m^{2}\right)=\frac{N_{c}}{16 \pi^{2}} \int_{1 / \Lambda^{2}}^{1 / \mu^{2}} \frac{\sqrt{\pi} d s}{s^{3 / 2}}(1+\operatorname{Erf}[\sqrt{s} v \cdot p]) \exp \left(-s\left(m^{2}-v \cdot p^{2}\right)\right)
$$

with $\operatorname{Erf}[a]=\frac{2}{\sqrt{\pi}} \int_{0}^{a} d x \exp \left(-x^{2}\right)$ being the Gaussian error function.

Finally, the Isgur-Wise function is calculated from the quark determinant by inserting an arbitrary current for a weak transition between heavy quarks of different velocity $\bar{Q}_{v^{\prime}}^{\prime} \Gamma Q_{v}$ with the result

$$
\xi(\omega)=\left.Z_{H}\left(\frac{2}{1+\omega}\left(I_{3}+v \cdot p I_{5}(\omega)\right)+m I_{5}(\omega)\right)\right|_{v p=\Delta M_{H}}
$$


The function $I_{5}$ of the momentum transfer $\omega=v \cdot v^{\prime}$ is expressed through the parameter integral

$$
I_{5}(\omega)=\int_{0}^{1} d y \frac{\partial}{\partial v \cdot p_{y}} \frac{I_{3}\left(v \cdot p_{y}, m^{2}\right)}{1+2 y(1-y)(\omega-1)}, \quad v \cdot p_{y} \equiv v \cdot p / \sqrt{1+2 y(1-y)(\omega-1)} .
$$

The normalization condition $\xi(1)=1$ comes out naturally as a Ward identity relating (8) and (11).

In ref. [8] we have discussed the Isgur-Wise function in the LME, which refers to the special case $v \cdot p=0$. In this case one has $I_{5}(\omega)=2 I_{2} r(\omega)$ with

$$
r(\omega)=\frac{\ln \left(\omega+\sqrt{\omega^{2}-1}\right)}{\sqrt{\omega^{2}-1}} .
$$

However, due to the introduced IR cut-off $\mu$ for the quark determinant (四) we can give up the LME around $v \cdot p=0$ and use eq. (11) for any value of $v \cdot p$.

Concerning the mass equation for the heavy mesons (8), let us point out that for $v \cdot p>m$ the integral $I_{3}$ generates non-physical behaviour in the IR region $(\mu \rightarrow 0, s \rightarrow \infty)$. This is easily understood, since through the optical theorem this is connected with an imaginary part reflecting a decay of a meson into real quarks which should be absent in a confining theory. For practical purposes for sufficiently small values $v \cdot p<m$, an expansion in $v \cdot p$ (LME) might be sufficient [8] . For the general case one better retains a finite value of $\mu$ which (in order to be on the safe side) we will chose in the following as $\mu=300 \mathrm{MeV}$. The basic assumption of chiral quark models like the one presented here is then to assume that the IR region of the quark dynamics excluded by the cut-off $\mu$ might be unimportant for the low-energy properties of mesons.

\section{Heavy meson spectrum and Isgur-Wise function}

Through eq. (8) the coupling constant $G_{3}$ is related to the heavy meson mass spectrum. For the light quark masses we will use the values $m_{u}=m_{d}=300 \mathrm{MeV}$ and $m_{s}=510 \mathrm{MeV}$. Let us first compare the numerical results for eq. (8) in contrast to the LME with $\mu=0$ in Fig. 2a. We observe significant differences between the two approaches for residual meson masses $\Delta M>m$. Furthermore, we can study the $\mu$-dependence of the heavy meson masses (see Fig. 2b). For $\mu \sim 300 \mathrm{MeV}$ we observe a reasonable mass-splitting between the parity conjugated fields $H, K$ of about $200-300 \mathrm{MeV}$.

In Table 1 we present the results in comparison with the LME, including the model results for the weak decay constant of the heavy meson given as $[8] \sqrt{M_{H}} f_{H}=\sqrt{Z_{H}} / G_{3}$.

\begin{tabular}{l|l|cc|cc|cc} 
& $G_{3}[\mathrm{GeV}]^{-2}$ & $\Delta M_{H}^{u}$ & $\Delta M_{H}^{s}$ & $\Delta M_{K}^{u}$ & $\Delta M_{K}^{s}$ & $f_{B}$ & $f_{B}^{s} / f_{B}$ \\
\hline \multirow{3}{*}{$\mu=300 \mathrm{MeV}$} & 8.2 & 200 & 255 & 500 & 685 & 135 & 1.04 \\
& 6.2 & 300 & 360 & 545 & 715 & 140 & 1.04 \\
& 4.5 & 400 & 475 & 590 & 750 & 150 & 1.04 \\
\hline LME [8] & 8.7 & 220 & 320 & - & - & 180 & 1.1
\end{tabular}

Table 1: Several heavy meson parameters in the extended NJL model (masses and decay constants in $[\mathrm{MeV}])$. 
a)

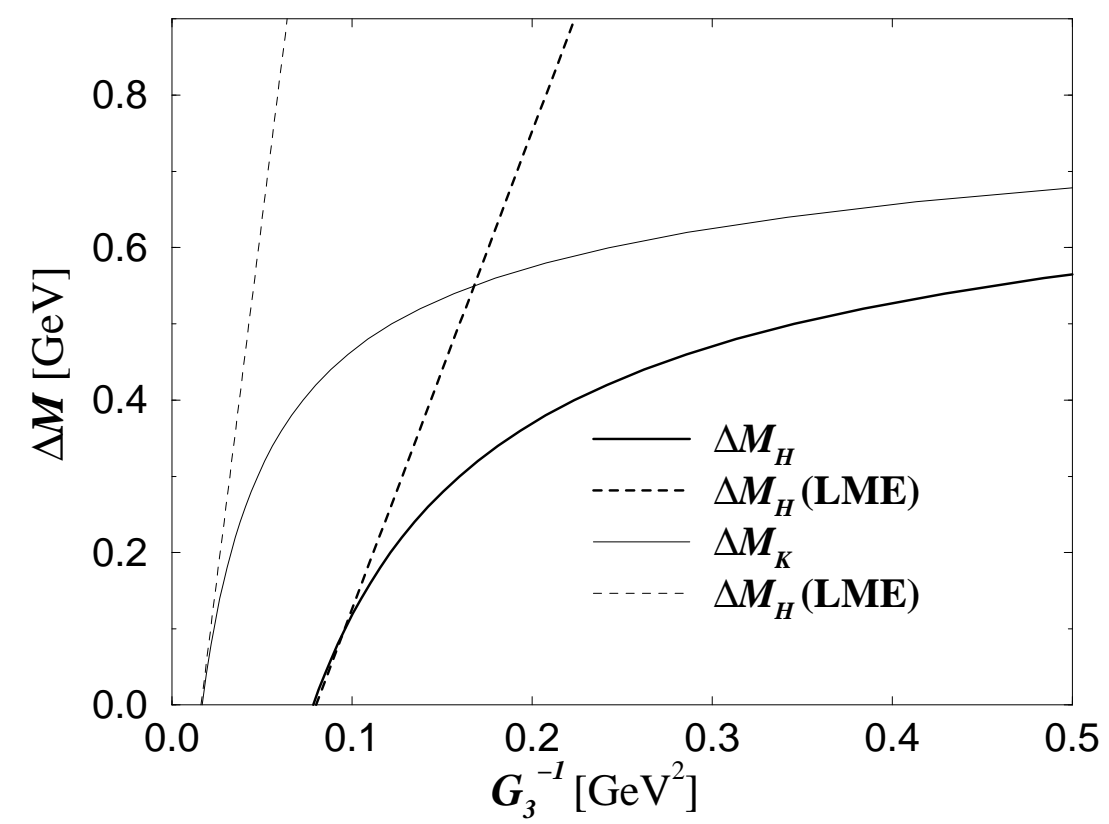

b)

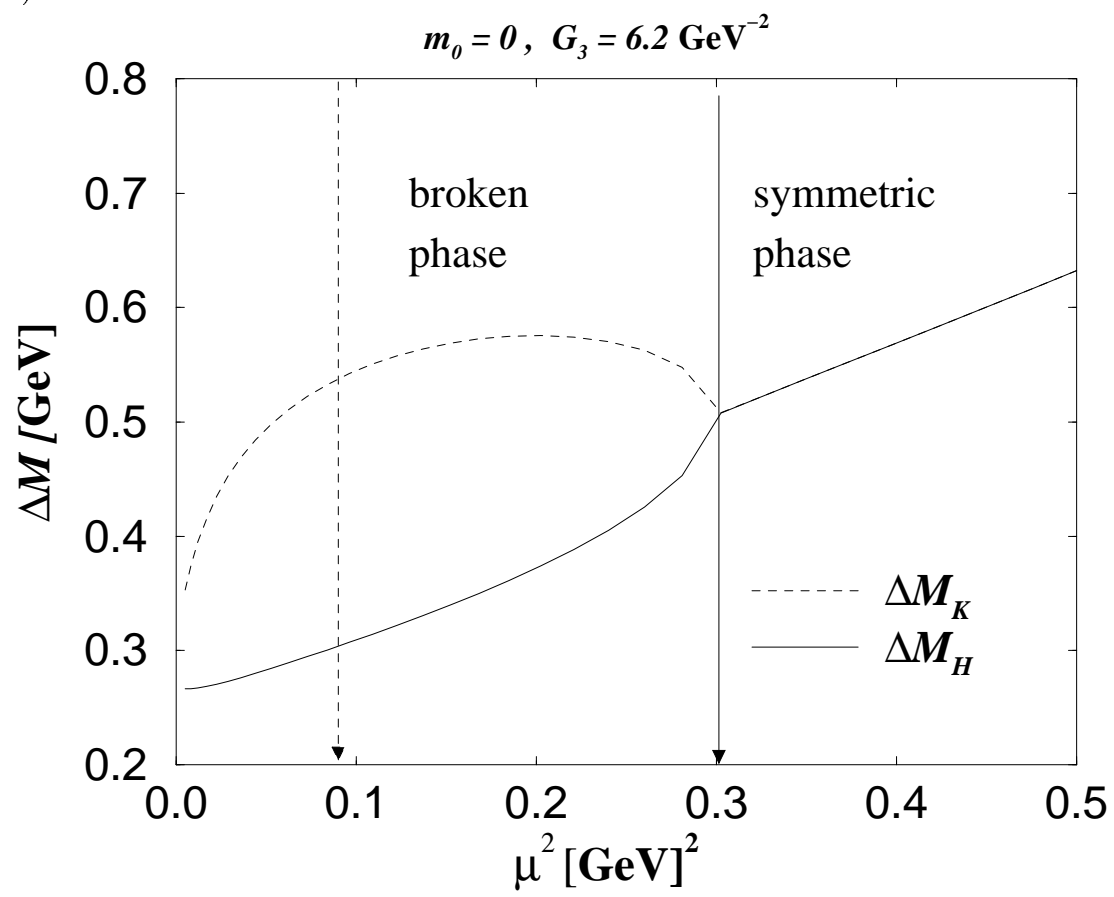

Figure 2: a) The behaviour of the residual masses $\Delta M_{H}$ (bold line) and of $\Delta M_{K}$ (thin line) as a function of $1 / G_{3}$ from eq. (8) for an infrared cut-off $\mu=300 \mathrm{MeV}$ compared to the LME with $\mu=0$ (dotted lines).

b) The behaviour of $\Delta M_{H}$ and $\Delta M_{K}$ in the chiral limit $m_{0} \rightarrow 0$ as a function of $\mu$ for a fixed value of $G_{3}=6.2 \mathrm{GeV}^{-2}$. Due to the restoration of chiral symmetry for $\mu \geq \mu_{c}$ the heavy meson fields $H, K$ fall into spin/parity quartets. 
Compared to the LME, the results for the heavy meson spectrum are improved, in the sense that they are leading to realistic masses even for the states with $J^{P}=\left(0^{+}, 1^{+}\right)$, while the results for $J^{P}=\left(0^{-}, 1^{-}\right)$are - as expected - only slightly effected. Note that all masses are now rather smooth functions of the coupling constant, which was not the case for the LME with $\mu=0$. The values for the weak decay constant lie on the lower side of the range obtained from lattice QCD [10], $f_{B}=170 \pm 55 \mathrm{GeV}$. The light flavor dependence can be studied by considering the $S U(3)_{F}$ masssplitting, which in our case is induced by different light quark masses $m_{u} \neq m_{s}$. Experimentally one has $M_{B_{s}}-M_{B_{u}} \simeq M_{D_{s}}-M_{D_{u}} \simeq 100 \mathrm{MeV}$ [11]. Our model predicts somewhat smaller values $\Delta M_{H_{s}}-\Delta M_{H_{u}} \approx 55-75 \mathrm{MeV}$. Note that this quantity only receives small $1 / m_{Q}$ corrections of the order $\left(m_{s}-m_{u}\right) / m_{Q}$.

Let us now concentrate on the Isgur-Wise function. In ref. [12] long-distance $\left(1 / m_{Q}\right)$ and short-distance $\left(\alpha_{s}\left(m_{Q}\right)\right)$ corrections have been estimated, which relate the Isgur-Wise function $\xi(\omega)$ to the experimentally accessible form factor(s) $\mathcal{F}\left(q^{2}\right)$. From this one of the CKM matrix elements has been estimated as $\left|V_{c b}\right|=0.039 \pm 0.002$. In particular, a phenomenological range for the slope parameter $\rho=\sqrt{-\xi^{\prime}(1)}$ is quoted, $0.84 \leq \rho \leq 1.00$, and a correlation between the slope and the curvature $c_{0}=\xi^{\prime \prime}(1) / 2$ is estimated as $c_{0} \simeq 0.72 \rho^{2}-0.09$ 13.

The result of the NJL model gives for different values of $v \cdot p=\Delta M_{H}$

$$
\begin{aligned}
\left.\rho\right|_{v p=0} & =0.67[8] \\
\left.\rho\right|_{v p=200 \mathrm{MeV}} & =0.77, \quad c_{0}=0.38 ; \\
\left.\rho\right|_{v p=300 \mathrm{MeV}} & =0.84, \quad c_{0}=0.49 ; \\
\left.\rho\right|_{v p=400 \mathrm{MeV}} & =0.94, \quad c_{0}=0.72 .
\end{aligned}
$$

The dependence on the external momentum is obvious. Values between $\Delta M_{H}=300 \mathrm{MeV}$ and $400 \mathrm{MeV}$ reproduce the phenomenological situation, and the values of $\Delta M_{H}, \rho$ and $c_{0}$ are positively correlated. Note that the inclusion of a finite curvature for the Isgur-Wise form factor is important in order to obtain an accurate fit to the data for the $B \rightarrow D \ell \nu$ decays which were used for the extraction of $\left|V_{c b}\right|$ [14].

Finally, we like to investigate the $\mu$-dependence of the shape parameters $\rho$ and $\xi^{\prime \prime}(1)$ which is of course rather stable for $v \cdot p \leq m$. For $v \cdot p>m$, our choice for the IR cut-off scale is justified, since for $\mu<300 \mathrm{MeV}$ the predictions for $\rho$ and $c_{0}$ become unreliable. This is illustrated in Fig. 3 for the two cases $v \cdot p=200 \mathrm{MeV}$ and $v \cdot p=400 \mathrm{MeV}$, respectively.

\section{Summary}

In this letter we have shown how the NJL model, which is phenomenologically successful at low meson energies, can be extended to higher energies. In particular we have shown that the appearance of quark thresholds, which plagues the application of the NJL model to higher external momenta can be avoided. This has been accomplished by introducing a finite adjustable IR scale when integrating out the quarks. We have found that for the spontaneous breaking of chiral symmetry the momentum regime between $\mu=300 \mathrm{MeV}$ and $\Lambda \approx 1.25 \mathrm{GeV}$ is responsible. Retaining a finite value for the IR scale (the choice $\mu \approx 300 \mathrm{MeV}$ has been favored by the data) prevents the quark determinant to suffer from unphysical quark-antiquark thresholds, which would otherwise occur in chiral quark models without explicit quark confinement.

Including heavy quarks in the infinite mass limit one then obtains a smooth dependence on the external momenta for the heavy meson observables, and a reasonable mass spectrum is found 
a)

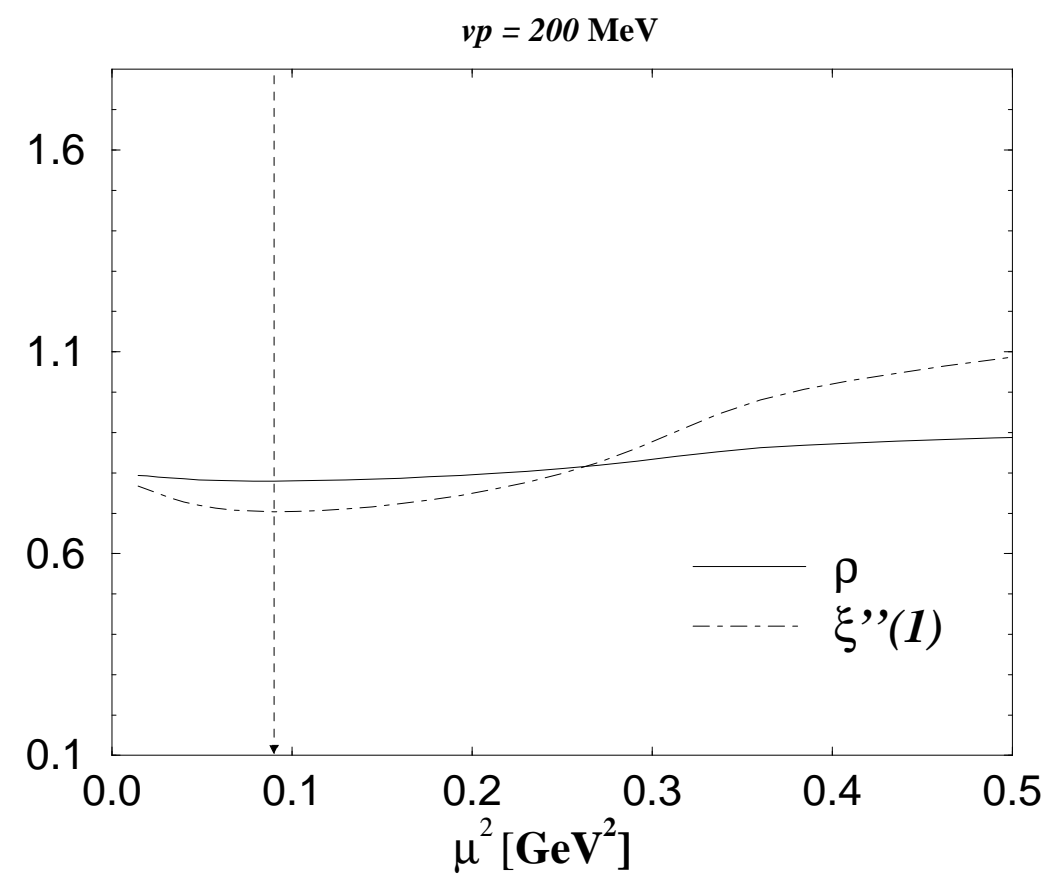

b)

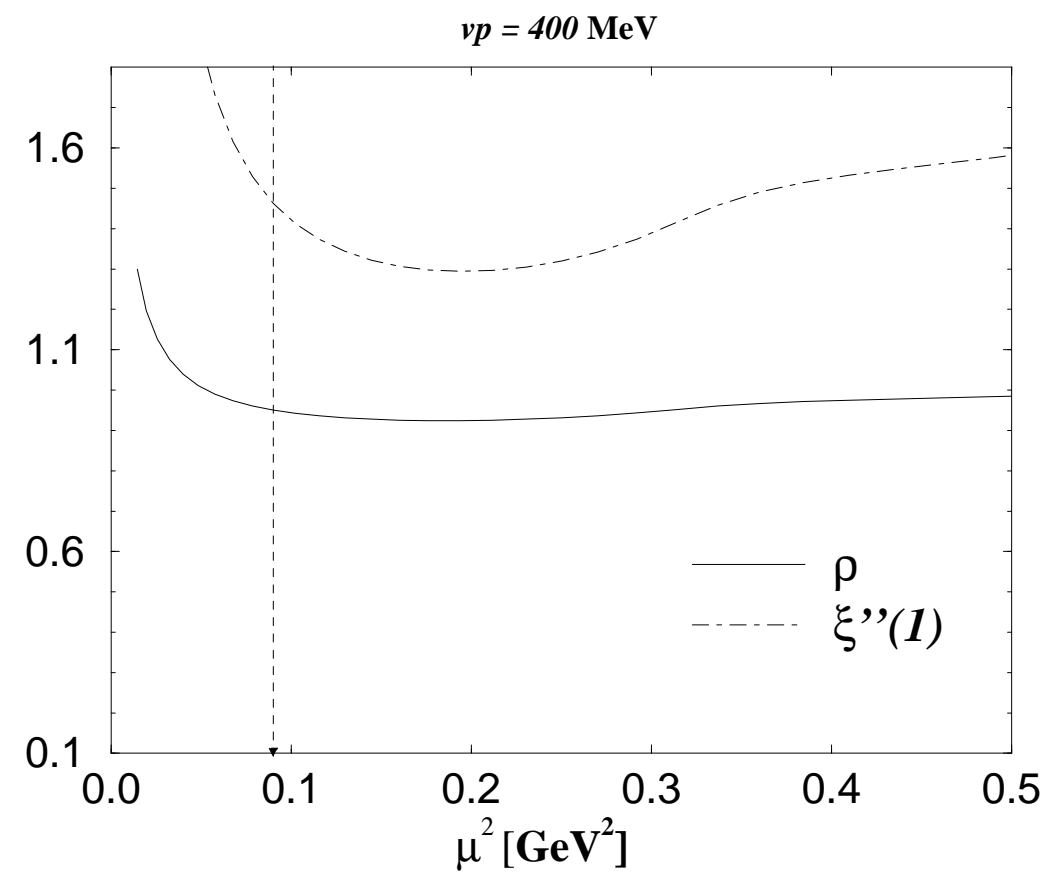

Figure 3: The behaviour of the shape parameters of the Isgur-Wise function, $\rho$ and $\xi^{\prime \prime}(1)$ as functions of the IR scale $\mu^{2}$ for a) $v \cdot p=200 \mathrm{MeV}$ and b) $v \cdot p=400 \mathrm{MeV}$. The vertical line denotes the choice $\mu=300 \mathrm{MeV}$. 
even for the heavy $J^{P}=\left(0^{+}, 1^{+}\right)$spin-symmetry multiplet. For the weak decay constants we found values of the order of $f_{B}=140 \mathrm{MeV}$.

For the shape parameters of the Isgur-Wise function we have found a non-negligible dependence on external momenta, such that the value of the slope parameter $\rho$ is positively correlated to the value of $\Delta M_{H}$. For $\Delta M_{H}=300(400) \mathrm{MeV}$ we found $\rho=0.84(0.94)$ which is consistent with the experimental and theoretical situation. We also give an estimate for the curvature at the nonrecoil point, $c_{0}=\xi^{\prime \prime}(1) / 2 \approx 0.49(0.72)$. At intermediate scales $\mu>300 \mathrm{MeV}$ the shape parameters develop only a weak dependence on $\mu$.

In future work our analysis can be helpful also for the investigation of higher resonances, like the heavy $J^{P}=\left(1^{+}, 2^{+}\right)$multiplet, within NJL-type quark models, where the dependence on external momenta is assumed to be even more important. Also the description of the higher excited light flavor states within the NJL model might be considerably improved. It can also be interesting to compare the approach presented here, with the results obtained in other relativistic quark models, which explicitly include confinement in a heuristic way.

\section{References}

[1] D. Ebert and H. Reinhardt, Nucl. Phys. B271 (1986) 188; for further references see D. Ebert, H. Reinhardt and M.K. Volkov, Prog. Part. Nucl. Phys. 33 (1994) 1.

[2] R.T. Cahill and S.M. Gunner, hep-ph/9601319.

[3] N. Isgur and M. Wise, Phys. Lett. B232 (1989) 113, Phys. Lett. B237 (1990) 527.

[4] A. F. Falk, Nucl. Phys. B378 (1992) 79.

[5] T. Mannel, W. Roberts and Z. Ryzak, Nucl. Phys. B368 (1992) 204.

[6] G. Burdman and J. F. Donoghue, Phys. Lett. B280 (1992) 287;

M. B. Wise, Phys. Rev. D45 (1992) R2188.

[7] R. Casalbuoni et al., hep-ph/9605342 (1996).

[8] D. Ebert, T. Feldmann, R. Friedrich and H. Reinhardt, Nucl. Phys. B434 (1995) 619.

[9] W. A. Bardeen, and C. T. Hill, Phys. Rev. D49 (1993) 409.

[10] H. Wittig, hep-ph/9606371, and refs. therein.

[11] L. Montanet et al. (Particle Data Group), Phys. Rev. D50 (1994), 1173.

[12] M. Neubert, hep-ph/9511409, and refs./ therein.

[13] I. Caprini and M. Neubert, hep-ph/9603414.

[14] M. Jimack (OPAL Coll.), Review of $V_{c b}$ Results, Talk presented at the 3rd German-Russian Workshop on Theor. Prog. in Heavy Quark Physics, Dubna 1996. 